\title{
Sciendo
}

RESEARCH PAPERS FACULTY OF MATERIALS

SCIENCE AND TECHNOLOGY IN TRNAVA

SLOVAK UNIVERSITY OF TECHNOLOGY

IN BRATISLAVA

2021, Volume 29, Number 49

DOI 10.2478/rput-2021-0022

\section{RELIABILITY ASSESSMENT OF THE SYSTEMS WITH SIMPLE INTERCONNECTIONS}

\author{
Eva LABAŠOVÁ ${ }^{1}$ \\ ${ }^{1}$ SLOVAK UNIVERSITY OF TECHNOLOGY IN BRATISLAVA, \\ FACULTY OF MATERIALS SCIENCE AND TECHNOLOGY IN TRNAVA, \\ INSTITUTE OF APPLIED INFORMATICS, AUTOMATION AND MECHATRONICS, \\ Ulica JÁnA BotTu 2781/25, 91724 TRNAVA, SLOVAK REPUBLIC \\ e-mail: eva.labasova@stuba.sk \\ Received 23 August 2021, Accepted 19 October 2021, Published 24 November 2021
}

\begin{abstract}
The paper deals with determining the final value of simple systems reliability. Simple systems, the systems with simple interconnections, can be divided into a sequence of series and/or parallel subsystems. The paper presents the basic functional relationships used to assess the reliability of simple systems, however, they are also essential for understanding more complex systems. The paper graphically shows and analyzes the final reliability of the systems depending on the arrangement of subsystems, and also those depending on the number of subsystems.
\end{abstract}

\section{Keywords}

Reliability function, series and parallel system, failure state and failure-free state

\section{INTRODUCTION}

Reliability is one of the basic and positive attributes of the quality of technical products and services. In daily life, we often encounter the usage of the adjective "reliable", especially in connection with advertising and marketing. In technical sciences, the term reliability expresses the ability of a product/object to perform the required functions in a specified time and under prescribed conditions.

A narrower definition of reliability in the electrical engineering industry [1] says: Reliability is a summary term used to describe availability and other factors that affect the availability. The reliability, maintainability and safety of maintenance are considered as the most important factors.

Generally, under the term reliability we understand the probability of failure-free operation during a specified period of time, given the object has been operated in accordance with the prescribed conditions. According to the above definition, reliability cannot be quantified. Yet, the factors of reliability can be quantified. One of the most important reliability features is the 
reliability function. Reliability can be characterized by several quantifiable indicators, such as e.g. failure rate, mean time to fault, mean time between faults, etc.

The basis for the calculations of reliability indicators is the transformation of the technological scheme into a reliability model. Each system can be divided into elements/subsystems that have different functional and technological interconnections. In reliability, most problems are solved by using models consisting of two-state elements (failure state and failure-free state). In general, the failure of a subsystem can be expected to affect the failure of other subsystems. In that case, we talk about two-state subsystems with dependent faults. The situation in which subsystem failures are independent of each other is more common. Block diagrams, fault trees and event trees are most often used to display two-state models.

The presented paper is focused on determining and comparing the reliability of simple twostate models with independent occurrence of faults. The target function will be the reliability function $R(t)$, which expresses the probability $P$ of survival [2]:

$$
R(t)=P(T>t)=R_{T}(t),
$$

where $T$ marks the random time of the system $(T=t+\Delta t, \Delta t$ is the length of the interval in the close vicinity of the selected value) and the failure-free system during the time interval $(0, t)$, resp. at time $t$ it is operable. The symbol $P$ marks the probability.

The reliability function expresses the probability of survival, and is an additional function to the cumulative distribution function $F_{T}(t)$. In the theory of reliability, the cumulative distribution function has the meaning of the failure function, resp. the function of the time to failure.

$$
\begin{aligned}
& R_{T}(t)=1-F_{T}(t) \\
& F_{T}(t)=P(T \leq t) .
\end{aligned}
$$

\section{CALCULATION OF RELIABILITY FUNCTION OF SIMPLE SYSTEMS USING BLOCK DIAGRAMS}

In the theory of reliability, the term of simple systems represents the systems that can be decomposed into a sequence of serially and/or parallel arranged subsystems (Fig. 1). We mark the reliability of subsystem with respect to the time $t r_{1}, r_{2} \ldots r_{i} \ldots r_{n}$, where $r_{i} \in(0,1)$.

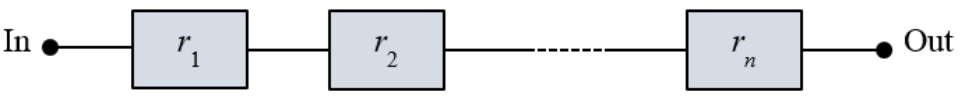

a)

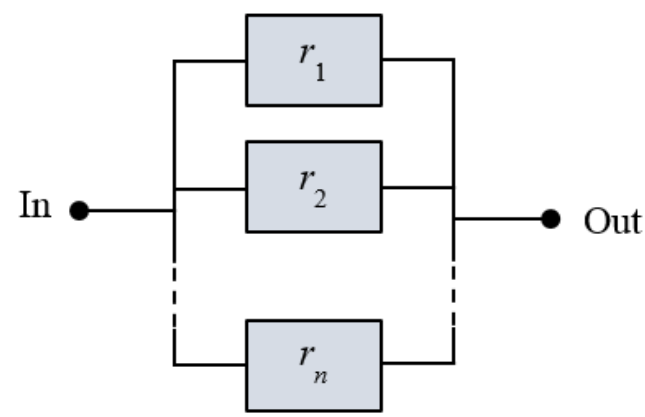

b)

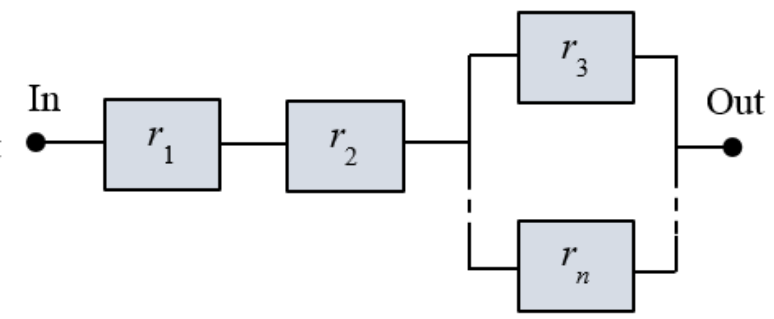

c)

Fig. 1 Block diagram of a system consisting of $n$ subsystems a) series arrangement, b) parallel arrangement, c) combined arrangement 


\section{THE SERIES SYSTEM}

The series system is in a failure-free state if all its subsystems are operable. In a series configuration, a failure of any subsystem leads to a failure of the entire system.

Let $T_{i}(i=1,2 \ldots, n)$ be the time to the failure of the single subsystems. Given none of the subsystems fails before time $t$, then and only then the system does not fail before time $t$. For independent failures, the probability of simultaneous occurrence of events is given by the product of the probabilities of single events.

$$
\begin{gathered}
R(t)=R_{T}(t)=P(T>t)=P\left(T_{1}>t \cap T_{2}>t \cap \ldots \cap T_{n}>t\right) \\
R(t)=R_{1}(t) R_{2}(t) \ldots R_{n}(t)=r_{1} r_{2} \ldots r_{n} .
\end{gathered}
$$

In general, the following applies to the final reliability $R_{S}$ of the series system [3]:

$$
R_{S}=\prod_{i=1}^{n} r_{i} .
$$

In reliability theory, the exponential distribution has exceptional position, which can be compared with the central position of the normal distribution, e.g. in quality control. In that case, the exponential distribution most often has the meaning of time to failure or time between failures. Instead of time, the fault event metric can also be given in the number of cycles, kilometers, etc. If the random variable is the time to failure, then the values of the random variable usually fall within the interval $\langle 0, \infty)$.

Considering the exponential distribution of the probability of a random variable $t$, the cumulative distribution function equals [4]:

$$
\begin{array}{ll}
F(t, \lambda)=1-e^{-\lambda t} \text { pre } t \geq 0 \\
F(t, \lambda)=0 & \text { pre } t<0
\end{array}
$$

$\lambda$ is the parameter of the scale for the exponential distribution. In that case, the following applies to the reliability function:

$$
R(t)=1-\left(1-e^{-\lambda t}\right)=e^{-\lambda t}
$$

The parameter $\lambda$ can also be interpreted as the failure rate $\lambda(t)$, which is constant for the exponential probability distribution.

$$
\lambda(t)=\frac{f(t)}{R(t)}=\frac{\lambda e^{-\lambda t}}{e^{-\lambda t}}=\lambda,
$$

where $f(t)$ is the probability distribution density for the exponential distribution. From a statistical point of view, the parameter $\lambda$ expresses the number of monitored events in the short consecutive time unit, and its unit is (time) ${ }^{-1}$, (number of cycles) ${ }^{-1}$ and so on.

For a series system, considering exponential distribution for each subsystem, the following applies:

$$
\begin{gathered}
R_{S}=\prod_{i=1}^{n} r_{i}=\prod_{i=1}^{n} e^{-\lambda_{i} t}=e^{-t \sum_{i=1}^{n} \lambda_{i}}=e^{-\lambda_{S} t} \\
\lambda_{S}=\sum_{i=1}^{n} \lambda_{i} .
\end{gathered}
$$

In the case of identical subsystems, if $r_{i}=r$, for the final reliability we get:

$$
R_{S}=r^{n}
$$

The following chart (Fig. 2) shows the dependence of the probability reliability of a system with series arranged identical subsystems on the number of subsystems. 
As can be seen from relation (10) and the chart in Fig. 2, final reliability of the series system depends on the number of subsystems that form the system, and also on the level of reliability of single subsystems. For subsystems with a low value of the probability of reliability, the final system reliability is near to 0 (Fig. $2 a$ ). From this point of view, it is more interesting to draw a graphical dependence of the final probability of system reliability when considering the subsystems with a high value of the failure-free probability (Fig.2b).

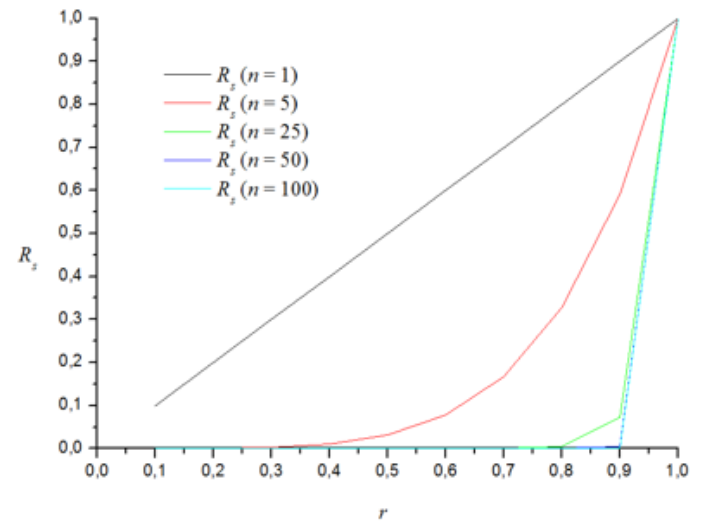

a)

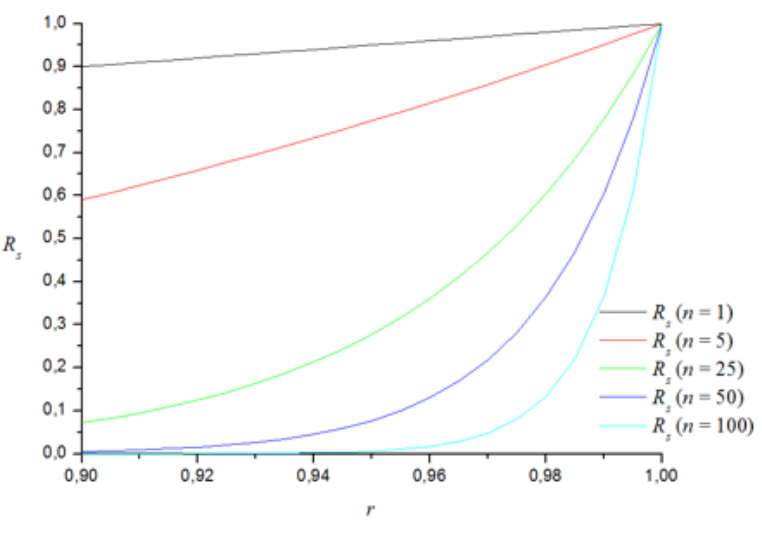

b)

Fig. 2 Probability reliability of series system $R_{s}$ depending on the number of identical subsystems $\left(r_{i}=r\right):$ a) for $\left.r \in(0 ; 1), b\right) r \in(0,9 ; 1)$

\section{THE PARALLEL SYSTEM}

The parallel system is in a failure state if all of its subsystems are no operable. In a parallel configuration, a failure of the last subsystem that was operational leads to the failure of the entire system.

Let $T_{i}(i=1,2 \ldots, n)$ be the times to the failure of the single subsystems. A failure of the system will occur before time $t$ only if a failure occurred in each of the subsystems. For independent failures, the probability $P$ of the simultaneous occurrence of events $T_{i} \leq t$ is given by the product of the probabilities of single events.

$$
\begin{gathered}
P(T \leq t)=P\left(T_{1} \leq t \cap T_{2} \leq t \cap \ldots \cap T_{n} \leq t\right)=P\left(T_{1} \leq t\right) P\left(T_{2} \leq t\right) \ldots P\left(T_{n} \leq t\right) \\
P(T \leq t)=F_{1}(t) F_{2}(t) \ldots F_{n}(t)=F_{T}(t) .
\end{gathered}
$$

The probability $P$ is expressed by the product of the cumulative distribution functions of the single subsystems. According to Equation (2), the following applies to the reliability of a parallel system:

$$
R_{T}(t)=1-F_{T}(t)=1-\left(1-F_{1}(t)\right)\left(1-F_{2}(t)\right) \ldots\left(1-F_{n}(t)\right) .
$$

In general, the following applies to the resulting reliability of the $R_{S}$ parallel system [3]:

$$
R_{S}=1-\prod_{i=1}^{n}\left(1-r_{i}\right) \text {. }
$$

In case of identical subsystems, if $r_{i}=r$, for the final reliability we get:

$$
R_{S}=1-(1-r)^{n} \text {. }
$$

For a parallel system considering exponential distribution for each subsystem, the following applies [4]:

$$
R_{S}=1-\prod_{i=1}^{n}\left(1-e^{-\lambda_{i} t}\right)
$$


The following chart (Fig. 3) shows the dependence of the probability of reliability of a system with parallel arranged identical subsystems on the number of subsystems.

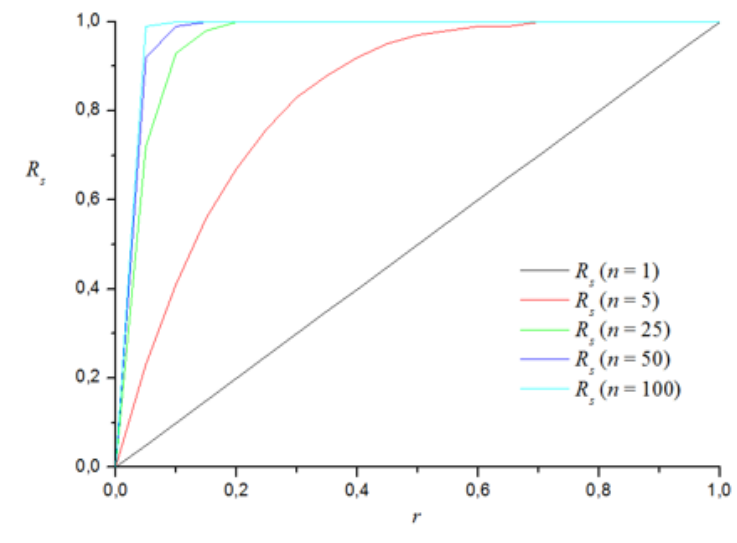

a)

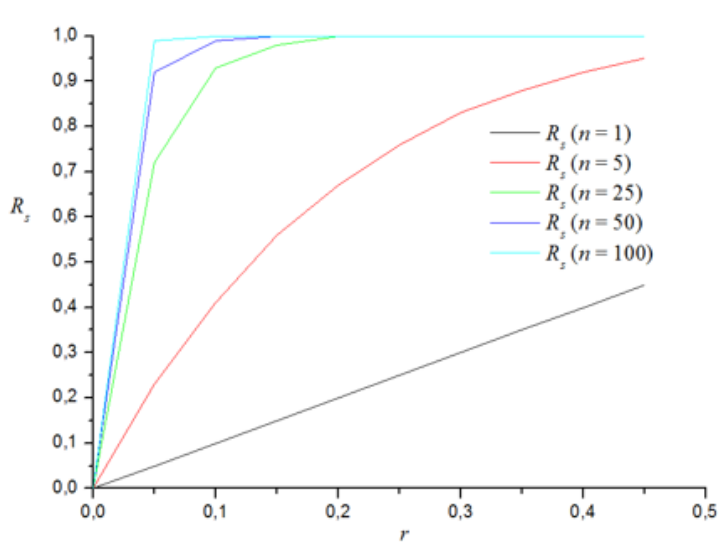

b)

Fig. 3 Probability of reliability of parallel system $R_{s}$ depending on the number of identical subsystems $\left.\left(r_{i}=r\right): a\right)$ for $\left.r \in(0 ; 1), b\right) r \in(0 ; 0,5)$

\section{THE COMBINED SYSTEM}

The combined system and its reliability is demonstrated by an example of a system consisting of 5 subsystems. The reliability is compared for 3 subsystems in series and 2 in parallel (Fig. 4a), and vice versa: 2 subsystems arranged in series and 3 in parallel (Fig. 4b).

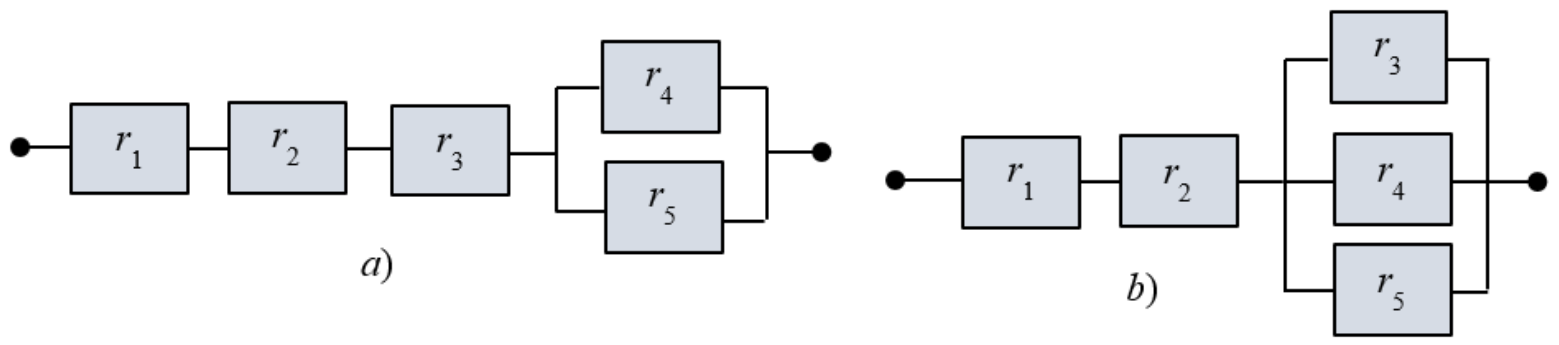

Fig. 4 Block diagram of a combined system consisting of 5 subsystems

Reliability of the entire system is given by the basic equations for the reliability of serial or parallel arrangement. For the system in Fig.4a), reliability equals:

$$
R_{\text {Sser }}=r_{1} r_{2} r_{3}\left[1-\left(1-r_{4}\right)\left(1-r_{5}\right)\right]
$$

Consider identical subsystems, after modification, we get:

$$
R_{\text {Sser }}=2 r^{4}-r^{5} \text {. }
$$

For the system in Fig.4b), reliability equals:

$$
R_{\text {Spar }}=r_{1} r_{2}\left[1-\left(1-r_{3}\right)\left(1-r_{4}\right)\left(1-r_{5}\right)\right] .
$$

Considering identical subsystems, after modification, we get:

$$
R_{\text {Spar }}=3 r^{3}-3 r^{4}+r^{5} \text {. }
$$

The following chart (Fig. 5) shows the dependence of the probability of reliability of a system with a predominant number of subsystems arranged in series (Fig. 4a) and a system with a predominant number of subsystems arranged in parallel (Fig. $4 b$ ). 


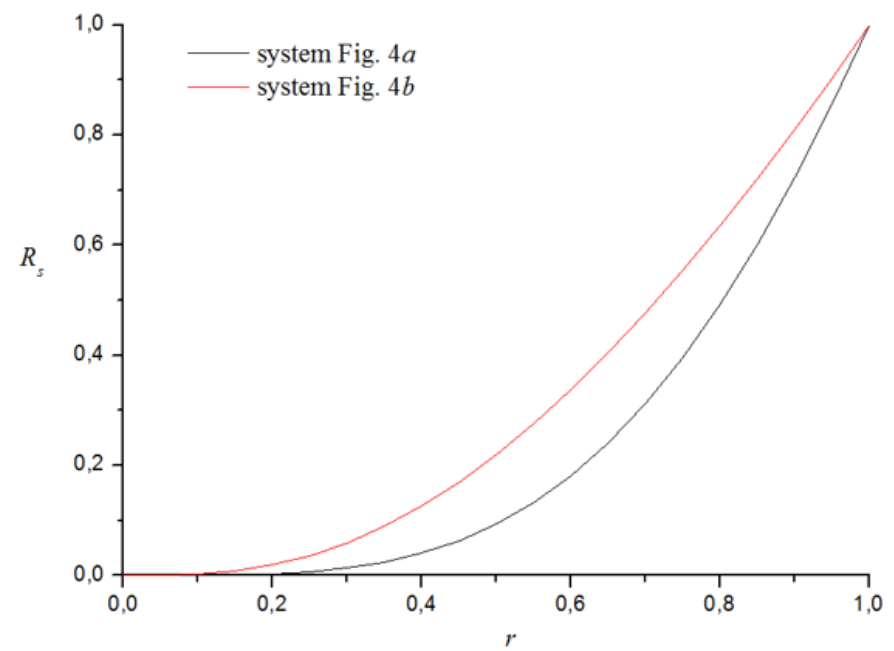

Fig. 5 Probability of fault-free state of the system $R_{s}$ depending on the number of identical subsystems arranged in series and in parallel

Sensitivity of the system to changes in the reliability of subsystems is expressed by partial derivatives. The subsystem with the highest sensitivity has the greatest impact on the reliability of the system.

For the system in FIG. 4a), relation (16) and considering identical subsystems, the following applies:

$$
\begin{gathered}
\frac{\partial R_{\text {Sser }}}{\partial r_{1}}=\frac{\partial R_{\text {Sser }}}{\partial r_{2}}=\frac{\partial R_{\text {Sser }}}{\partial r_{3}}=2 r^{3}-r^{4} \\
\frac{\partial R_{\text {Sser }}}{\partial r_{4}}=\frac{\partial R_{\text {Sser }}}{\partial r_{5}}=r^{3}-r^{4} .
\end{gathered}
$$

From relations (20), (21) it is obvious that the sensitivity of the system $\left(R_{S s e r}\right)$ to the change $r_{1}, r_{2}, r_{3}$ is higher compared to the change $r_{4}, r_{5}$ because:

$$
2 r^{3}-r^{4}>r^{3}-r^{4}
$$

For the system in FIG. 4b), relation (18) and considering identical subsystems, the following applies:

$$
\begin{gathered}
\frac{\partial R_{\text {Spar }}}{\partial r_{1}}=\frac{\partial R_{\text {Spar }}}{\partial r_{2}}=3 r^{2}-3 r^{3}+r^{4} \\
\frac{\partial R_{\text {Spar }}}{\partial r_{3}}=\frac{\partial R_{\text {Spar }}}{\partial r_{4}}=\frac{\partial R_{\text {Spar }}}{\partial r_{5}}=r^{2}-3 r^{3}+r^{4} .
\end{gathered}
$$

From relations (23), (24) it is obvious, that the sensitivity of the system $\left(R_{\text {Spar }}\right)$ to the change $r_{1}, r_{2}$, is higher compared to the change $r_{3}, r_{4}, r_{5}$ because:

$$
3 r^{2}-3 r^{3}+r^{4}>r^{2}-3 r^{3}+r^{4}
$$

\section{CONCLUSION}

The method of block diagrams of reliability is relatively simple to process; for less complex systems, it does not require software support, and is therefore relatively widely used. The aim of the paper was to provide the basic overview for determining reliability of simple systems. At the same time, the objective was to compare reliability of the systems with serial and parallel arrangement. We assumed that the individual subsystems were two-state and independent (the state of any system does not affect the state of other subsystems in any way).

Final reliability of the systems depends on the number of subsystems that make up the system, and also on the level of reliability of the individual subsystems. In the case of series 
arranged subsystems, the value of the probability of system reliability decreases with increasing number of system elements. In a series configuration, a failure of any subsystem leads to a failure of the entire system. In the case of subsystems arranged in parallel, the value of the reliability probability of the system increases with the increasing number of system elements. In a parallel configuration, the failure of the last subsystem that was functional leads to the failure of the entire system.

In combined systems, which can be decomposed into a combination of subsystems arranged in series and in parallel, the reliability depends on the specific number and arrangement of the subsystems. The reliability of the system depends on the number of elements arranged in series and in parallel. In a combined system configuration, failure of any subsystem in a series arrangement results in failure of the entire system or if all subsystems arranged in parallel fail. The probability of failure is therefore lower (or the probability of reliability is greater) if a parallel arrangement of subsystems prevails in the system.

\section{Acknowledgement}

This publication was supported by the Scientific Grant Agency of the Slovak Republic VEGA under the grant No. 1/0144/20.

\section{References}

[1] STN IEC 60050-191, 1993. International Electrotechnical Vocabulary. Chapter 191: Dependability and quality of service.

[2] JANÍČEK, F., KOVÁCS, Z. 2009. Reliability in electro energetics. ISBN 978-80-89402-12-0

[3] MYERS, A., 2010. Complex system reliability. ISBN 978-1-84996-413-5

[4] VRBAN, A., 2007. Reliability of management systems. ISBN 978-80-8096-010-0

\section{ORCID}

Eva Labašová 0000-0001-9055-1233 